Acta Crystallographica Section D

\section{Biological Crystallography}

ISSN 0907-4449

\section{Elspeth F. Garman* and Robin Leslie Owen}

Laboratory of Molecular Biophysics, Department of Biochemistry, University of Oxford, Oxford OX1 3QU, England

Correspondence e-mail: elspeth@biop.ox.ac.uk

\title{
Cryocooling and radiation damage in macromolecular crystallography
}

Advances in cryocrystallographic techniques for macromolecular crystallography have been intimately intertwined with efforts to reduce the deleterious effects of X-ray damage inflicted during the collection of diffraction data. A brief overview of cryomethods and their rationale is given. This is followed by a summary of our current limited understanding of radiation damage in cryocooled crystals, investigations aimed at minimizing its effects and finally some developments which actually utilize it both for phasing and to extend structural knowledge.

\section{Introduction}

The macromolecular cryocrystallographic techniques developed over the last 20 years have had a huge impact on protein crystallography and have also allowed our brightest synchrotron sources to be utilized efficiently. Most macromolecular crystals held at room temperature during data collection lose their order after only a few seconds in an unattenuated thirdgeneration synchrotron source X-ray beam. For a crystal held at $100 \mathrm{~K}$, a much longer total X-ray exposure can be tolerated without severely affecting the diffraction quality, so usually one or more complete data sets can be collected from a single crystal. Methods for cryocooling have thus become well established and widely disseminated, with over $90 \%$ of biological crystallographers currently collecting their data at around $100 \mathrm{~K}$. Research in this area is now largely directed at understanding the reasons why certain protocols for cooling are on average more successful than others.

Although the problem of radiation damage is much reduced if the sample is held at $100 \mathrm{~K}$ during data collection, at thirdgeneration synchrotron sources there is commonplace observation of decreasing diffraction quality as the cumulative $\mathrm{X}$-ray exposure increases. Damage occurring to cryocooled $(100 \mathrm{~K})$ crystals was in fact first noted at a second-generation source (Gonzales et al., 1992). These observations have resulted in a recent research effort to gain some understanding of the characteristics of radiation damage at $100 \mathrm{~K}$ so that strategies to minimize it might be devised, data might be corrected for it, and it might be established if its effects could be used positively to gain structural information. This research is still in its infancy; the experiments are painstaking and time consuming, but the investigations have made some progress over the last five years.

The first systematic study of the effect of X-rays on protein crystals was carried out at room temperature in 1962 by Blake and Phillips on crystals of myoglobin. They observed that damage was proportional to dose and that each $8 \mathrm{keV}$ X-ray photon absorbed by the crystal appeared to disrupt $\sim 70$
Received 14 June 2005 Accepted 21 October 2005 
molecules and somewhat disorder another 90 . They proposed an empirical model for diffraction decay which could not account for all the changes in diffraction intensities that they observed: 'there are, however, some small but significant changes in the diffracted intensities which may indicate structural effects of the irradiation' (Blake \& Phillips, 1962). This postulate was subsequently confirmed for cryocooled crystals held at $100 \mathrm{~K}$ (Burmeister, 2000; Ravelli \& McSweeney, 2000; Weik et al., 2000). For room-temperature data collection, broken disulfides were observed in electrondensity maps for crystals of ribonuclease by Burley, Petsko and Ringe, this was noted in Helliwell (1988) who observed similar effects in crystals of insulin.

The benefit of cooling protein crystals during data collection was quantified in 1970 by Haas and Rossmann, who reported a tenfold reduction in the rate of intensity loss of two reflections in lactate dehydrogenase crystals soaked in $3 M$ sucrose when they compared $198 \mathrm{~K}$ with room-temperature measurements (Haas \& Rossmann, 1970). A general method of exchanging mother liquor with an aqueous/organic mixture of high organic solvent concentration prior to crystal cooling was presented by Petsko (Petsko, 1975), and in the 1980s Hope encouraged macromolecular crystallographers to adopt the oil and glass spatula techniques of crystal mounting used for small-molecule crystallography (Hope, 1988). The suggestion of using a small thin loop and surface-tension forces to hold the crystal (Teng, 1990) and the commercial availability of a reliable and easy-to-use cryostat (Cosier \& Glazer, 1986) were both pivotal advances in the development of macromolecular cryocrystallography, and led to its widespread application and use. This is mainly because of the much reduced rate of radiation damage but also because of several other advantages: usually a whole data set can be collected from one crystal resulting in higher quality data, the loop mounting technique is much easier, as well as being less harmful to fragile crystals than the traditional room temperature capillary methods, crystals can be flash-cooled and stored under liquid nitrogen when they are in peak condition, and new types of experiments are now possible such as cryotrapping of reaction intermediates. Cryocooling protocols are now an essential step in data collection from most crystals and have had a major impact on the quality and number of biological structures being obtained.

However, the increasingly high fluxes offered by thirdgeneration synchrotron sources have resulted in radiation damage being observed even when the crystals are held at around $100 \mathrm{~K}$. This damage manifests itself in a number of different ways including; decreasing diffraction power, increases in unit-cell volume, and specific structural damage to covalent bonds. The latter two phenomena result in creeping non-isomorphism as the experiment proceeds, which can cause the MAD method of structure solution to fail. While damage to specific sites can be used to obtain phase information (Ravelli et al., 2003), the observation that active sites in protein molecules seem particularly sensitive to change means that radiation damage has once again become a relevant concern for all structural biologists.
In this paper we summarize the widely used cryocooling techniques and the current state of our understanding of radiation damage in macromolecular cryocrystallography. For fuller accounts of cryocrystallographic methods and hardware, the reader is referred to the comprehensive accounts that are already in the literature and which will not be repeated here (Garman \& Schneider, 1997; Rodgers, 1997; Parkin \& Hope, 1998; Garman, 1999b; Hope, 2001; Rodgers, 2001; Garman\& Doublié, 2003; Pflugrath, 2004). Brief comments on the current status of radiation-damage studies can also be found in Garman \& Nave (2002) and Nave \& Garman (2005), which are the introductions to two issues of the Journal of Synchrotron Radiation containing papers from the Second (2001) and Third (2003) International Workshops on X-ray Damage to Biological Crystalline Samples, respectively.

\section{How do we cool?}

The various practical aspects of cryotechniques have become routine in macromolecular crystallography, but they often become modified and non-optimal with time as they are handed down, largely anecdotally, to new researchers. In most cases this gives reasonable results, but occasionally it fails completely, and then the basic techniques have to be revisited in order to find a suitable way forward. The various procedures recommended in the papers listed above have a rationale based on finding the cryoprotocol which will ultimately give the optimum diffraction data quality (Garman, 1999b). Their application ensures the best chemical conditions for the crystal (e.g. cryoprotectant composition and concentration, minimum osmotic shock) and the least damaging manipulation and flash-cooling procedure, in order to retain the physical state of the crystal. Careful attention to these two factors can significantly improve the quality of a data set and thus make subsequent structure solution and refinement more straightforward.

Once cooled, the crystal must be kept below the glass transition temperature of the cryobuffer at or below $155 \mathrm{~K}$ (Weik, Kryger et al., 2001) (increased from the $136 \mathrm{~K}$ of pure water by the mother liquor constituents) at all times (except if annealing - see below).

\subsection{Cryoprotection}

The basic tenet of cryocrystallographic protocols is that on cooling, the formation of crystalline ice within the sample must be avoided since it both disrupts internal order (owing to expansion on formation) and also interferes with the diffraction pattern from the protein. Thus, the crystal must be cooled so fast that the water in the solvent channels is in the vitreous rather than the crystalline state at the end of the procedure (Fig. 1). For pure water, vitrification of micrometre sized drops can be achieved with cooling times of the order of $10^{-5} \mathrm{~s}$ (Johari et al., 1987): clearly an impossible requirement for solvent in interstitial sites in crystals. This problem can be overcome by replacing some of the water in the mother liquor with a cryoprotectant agent ('antifreeze') which effectively 
Table 1

Possible strategy for finding an appropriate cryoprotectant buffer.

\begin{tabular}{|c|c|}
\hline Major component of mother liquor & Suggested cryoprotectant agents/strategy \\
\hline All components & $\begin{array}{l}\text { Add } 15-25 \% \text { glycerol (works in around two thirds of cases). } \\
\text { Drag through oil (e.g. paraffin, paratone-N or NVH) } \\
\quad \text { (Riboldi-Tunnicliffe \& Hilgenfeld, 1999) }\end{array}$ \\
\hline $\mathrm{PEG}<4 \mathrm{~K}$ & Increase PEG, add small molecular weight PEGs \\
\hline $\mathrm{PEG}>4 \mathrm{~K}$ & $\begin{array}{l}\text { Add small molecular weight PEGs ( } N B \text { large MW PEGs are } \\
\text { poor cryoprotectants and are very viscous) }\end{array}$ \\
\hline Crystal screens solutions I (14) & $\begin{array}{l}\text { Glycerol concentrations required given in Garman \& Mitchell } \\
\text { (1996) }\end{array}$ \\
\hline Crystal screen solutions I and II (14) & $\begin{array}{l}\text { PEG400, ethylene glycol, glycerol and } 1,2 \text { propanediol } \\
\text { concentrations required given in McFerrin \& Snell (2002) }\end{array}$ \\
\hline MPD & Increase MPD concentration \\
\hline $\begin{array}{l}\text { Salt ( } N B \text { low-salt concentration requires } \\
\text { a greater concentration of cryoprotectant } \\
\text { than high salt) }\end{array}$ & $\begin{array}{l}\text { (a) Add MPD and/or ethylene glycol or glycerol or a mixture } \\
\text { of sugars such as } 15 \% \text { trehalose }+15 \% \text { sucrose } \\
\text { (b) Increase concentration /add salt (e.g. } 8 \mathrm{M} \mathrm{Na} \text { formate or } \\
\text { malonate) (Rubinson } \text { et al., } 2000) \\
\text { (c) Exchange salt for organic solvent (Wierenga et al., 1992) }\end{array}$ \\
\hline When all else fails... & $\begin{array}{l}\text { Try } 20 \% \text { L-(+)-2,3-butanediol (Wynne et al., 1999; Tucker, } \\
\text { 2005) }\end{array}$ \\
\hline
\end{tabular}

extends to $1-2 \mathrm{~s}$ the time available for the cooling process to result in vitrification as opposed to crystallization. There are two types of cryoprotectant agent commonly used: $(a)$ those such as glycerol which penetrate into solvent channels and (b) oils with which the crystal is coated (Riboldi-Tunnicliffe \& Hilgenfeld, 1999). A survey of cryoprotectant useage (Garman \& Doublié, 2003) showed that around $50 \%$ of researchers use glycerol, the majority adding between 20 and $30 \%(v / v)$. This by no means implies that glycerol is always best; it is often just the most convenient, and no subsequent optimization takes place because it gives adequate results. Oils do not penetrate into the crystal but provide a barrier between the surface of the crystal and the air, and best results are obtained if as much mother liquor is removed as possible. The crystal can then be covered with a protective layer of oil to avoid dehydration during its transfer to cryogen, although this procedure is not always successful in avoiding ice.

However, a second requirement for success in cryocooling is imposed during soaking; the chosen cryobuffer should not degrade the crystal by either surface attack or osmotic shock. Thus, when deciding which cryoprotectant agent to try, the components of the mother liquor should first be considered. If a cryoprotectant agent is already present at low concentration, the concentration can often be increased without deleterious effects. Table 1 gives a general idea of where to start in selecting a cryoprotectant agent. A benign agent will neither attack the crystal surface (this ultimately results in the crystal dissolving), nor cause its surface to increasingly resemble crazy paving because of osmotic shock.

The putative cryobuffer is first mounted alone in the loop (i.e. no crystal) and flash-frozen for a diffraction test. A glassy looking result is a necessary but not sufficient condition for adequate cryoprotectant agent concentration: for instance a water:glycerol mixture of $60 \%: 40 \%$ looks glassy but gives an ice diffraction ring at $3.67 \AA$ (see Fig. 2), with a signal/noise $(\mathrm{S} / \mathrm{N})$ ratio of 2 , and it is necessary to go to a $55 \%: 45 \%$ mixture to reduce this to 1.5. Thus, a diffraction test allows the optimum concentration to be found. Since the cryobuffer concentration is often slightly diluted by the addition of the crystal and its surrounding mother liquor; a safe strategy is to increase the initial concentration by $2-5 \%$.

Once a suitable cryobuffer has been identified, the overall cryoprotocol can be optimized along the lines shown in Fig. 3, which gives a possible strategy. In the experience of the authors, the single most common reason for failure (i.e. diffraction not adequate for the planned experiment) in cryocooling is that the cryobuffer has been made by diluting

\section{Figure 2}

Optimization of cryobuffer. Diffraction patterns illustrating the effect of adding increasing concentrations of glycerol to water. For $100 \%$ water, discrete reflections from individual ice crystals are observed. As the concentration of glycerol is raised, the diffraction shows typical sharp powder pattern rings from the ice crystallites, becoming a single sharp ring and then a diffuse ring with gentle gradients on both the high- and low-resolution sides. Adequate cryoprotection has been achieved with $45 \%: 55 \%(v / v)$ glycerol:water. The resolutions at which ice reflections appear are also tabulated. 


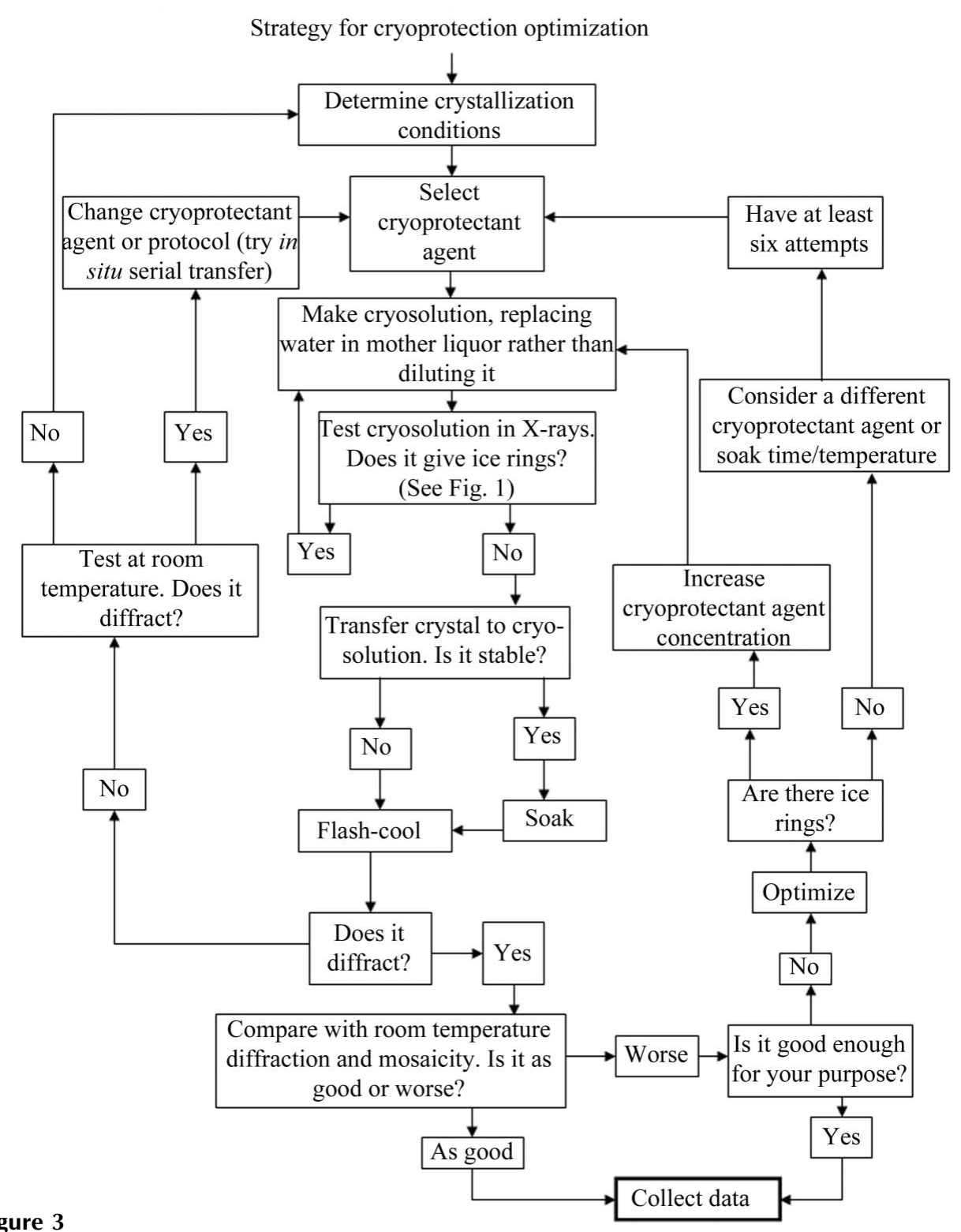

Figure 3

Flow diagram showing a possible strategy for optimizing the cryoprotocol. can then be fished directly from the mother liquor for cooling. There are now several commercial crystallization screens available in which the solutions are already fully cryoprotected, and this is an increasing trend.

It is believed that the optimum cryobuffer for a particular crystal species is the one which contracts on cooling by the same or similar proportion to the crystal lattice, so that the minimum of disruption to the crystal order will be inflicted (Kriminski et al., 2002).

\subsection{Equipment}

Some equipment and instruction in its use is necessary before starting to flash-cool crystals: a reliable cryostat delivering a stream of gaseous nitrogen at around $100 \pm 1.0 \mathrm{~K}$ with an outer sheath of roomtemperature dry air or nitrogen, a magnet which fits into the $3 \mathrm{~mm}$ diameter hole in the top of a goniometer head, some tophats (cryocaps), pins and rayon/ nylon loops, cryovials, cryocanes, cryosleeves to secure the vials on the canes for transport, and lastly but not least, handling tools. This equipment is well described in the literature, and available from several vendors. However, it is still far from the mother liquor with cryoprotectant agent, rather than by replacing the water in it. The dilution method, although more convenient, lowers the concentration of the components of the mother liquor, and it is thus not surprising that the crystals often do not survive the experience.

In cases where difficulty is experienced in finding suitable cryoconditions, the effects of temperature and osmotic shock should be investigated. For instance, a $277 \mathrm{~K}$ soak and/or in situ serial transfer could be tried, or more exotic cryoprotectants such as mixtures of light and heavier sugars could be used, e.g. $15 \%$ trehalose $+15 \%$ sucrose is particularly good for salt-based crystal buffers. Also mixtures of different cryoprotectant agents have been used, or a cryobuffer followed by immersion in oil (Kwong \& Lui, 1999).

The best scenario is when the crystal mother liquor already contains a high enough concentration of cryoprotectant agent for flash-cooling so that no more need be added. The crystal standard, and this is well illustrated in Fig. 4, where four different cryopin/cryocap arrangements are shown. Efforts by the SPINE collaboration to arrive at a concensus common design of cryopins for Europe have resulted in the development of the pin in Fig. 4(d).

Recent developments have provided experimenters with two more robust alternatives to the commercially available rayon loops. These are the so-called 'litho-loops ${ }^{\mathbf{1}}$ which are etched from mylar film and come in a range of circular and elliptical sizes, and the 'micro-mounts', (Thorne et al., 2003) which are fabricated from patterned and shaped thin polyimide film. Both of these represent significant improvements, since they do not unwind with time, they are precisely the size they claim to be and they are more rigid than fibre loops.

\footnotetext{
${ }^{1}$ Molecular Dimensions, http://www.moleculardimensions.com

${ }^{2}$ MiTeGen, http://www.mitegen.com.
} 


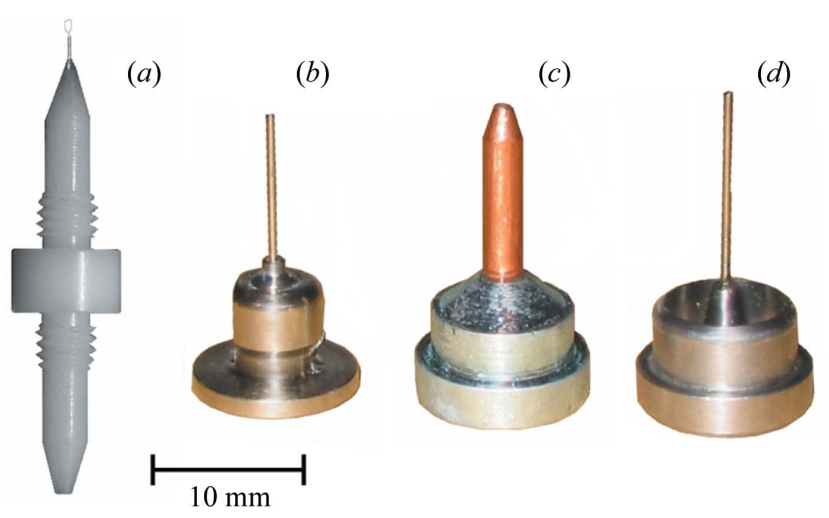

Figure 4

A gallery of some cryopins currently available. (a) Pins used at SPring-8, Japan (Ueno et al., 2004), (b) Oxford Cryosystems nickel pin, (c) Hampton Research brass pin on stainless base, and $(d)$ SPINE (Structural Proteomics in Europe) pin machined for use with robotic sample changers and having a data matrix barcode on its base to allow tracking of a sample through the experimental structure-determination process.

As with the cryoprotection, there is a single common and easily rectified problem which often results in a failed experiment: the pins should all be a standard known length that will keep crystals in the centre of the cryostat nitrogen stream at all times, even when rotated on a removable arc (see below). Ideally all of the pins and cryocaps in the laboratory should conform to the standard length. This will save a great deal of aggravation in the long run. Before stream-cooling, a standard length pin should be pre-aligned on the goniometer before the crystals are opened and manipulated.

\subsection{Transfer of crystal to cryobuffer}

If the crystals require gentle surgery to remove 'skin' or separate clusters before being mounted in a loop, acupuncture needles are very useful for this purpose since they are thin, slightly flexible, and better than syringe needles as there is no capillary action to remove liquid and beach the crystals. The needles can be reused many times.

For a crystal grown in the presence of an adequate concentration of cryo-agent, the transfer step is unnecessary and it can be fished straight out of the drop into the cryogen, thereby minimizing handling. As a general rule, the less a crystal is manipulated the better. Handling can cause damage to the crystal surface as well as dehydration, especially if the crystal is transferred in the loop through air several times. These traumas invariably result in an increase in the mosaicity of the crystal.

There are broadly three ways to transfer a crystal from its growth drop into the cryobuffer: soaking, vapour-pressure equilibration and dialysis. For the soaking method used in the vast majority of cases, the crystal is transferred from its growth drop with a loop (or for fragile crystals, a suction device made from a pulled Pasteur pipette, a piece of flexible tube and a small syringe) straight into the final concentration of cryobuffer. It is left there for anything from $1 \mathrm{~s}$ ('quick dip') to minutes or days, depending on whether or not it is stable. A soak time of between 1 and $3 \mathrm{~min}$ is the general norm. A kinder strategy for the crystal is to transfer it first into a small volume of mother liquor, and then to pipette a volume of the cryobuffer onto it, mix with the pipette tip, remove an equal volume and repeat. This method can also be used for serial increase of the cryobuffer concentration, starting with say $10 \%(v / v)$ added, mixed and removed twice, moving on to $20 \%$ and so on until the required concentration is reached. $40 \%$ can be reached in about $3 \mathrm{~min}$ in this way, and in a significant number of cases this strategy has been found to produce better results (higher resolution diffraction and lower mosaicity) than quick dips or serial transfers where the crystal is moved between drops of increasing concentration. It involves a lot less crystal manipulation and a gentler gradient to the cryo-agent concentration increase (Garman, 1999b).

For vapour-pressure equilibration, the cover slip with the hanging drop containing the crystals is sealed over a volume of cryobuffer in the bottom of a tray overnight, to allow equilibration of the vapour components. The crystal is then given a quick soak as before. This method has been found to be less invasive by some researchers, who always use it (Gamblin, 2005).

In difficult cases, the cryobuffer can be dialysed slowly into the crystal. This method is not often used, but can be something to try for cases where a benign cryobuffer cannot be found, and the use of oil fails.

The crystal is now ready to be fished out of the cryobuffer and flash-cooled straight into liquid cryogen (which should be positioned next to the microscope) or in a gas stream (move the microscope as near to the stream as possible). It is a good idea to practice fishing some non-essential crystals before trying the precious ones. In most cases, a size of loop matched to the crystal size should be chosen, as this will minimize the liquid volume (i.e. reduce $\mathrm{X}$-ray scatter and thus maximize signal/noise) and small crystals are very hard to 'catch' in a large loop as they just go through it. To fish, the liquid is first agitated in order to lift the crystal from the bottom of the drop. If it is stuck, an acupuncture needle can be gently used to free it. The loop is brought up to the crystal from the side with its plane vertical, so that it encloses the crystal. The loop is then pulled upwards so that its edge breaks the surface tension of the drop. Leaving the plane of the loop perpendicular to the surface of the liquid minimizes the forces on the crystal and also minimizes the thickness of the liquid film.

The two pivotal times in this procedure are the time it takes to transfer the crystal from the cryobuffer to the cryogen, and the time the crystal takes to be cooled below the glass transition at around $155 \mathrm{~K}$. For optimum results, both these times should be as short as is practicable.

\subsection{Cryogen}

Out of all diffraction experiments reported in Acta Crystallographica Section D in 2002, 60\% of crystals were flashcooled in a nitrogen gas stream $(\sim 100 \mathrm{~K}), 37 \%$ in liquid nitrogen $(77 \mathrm{~K})$, and $3 \%$ in liquid ethane or propane: experimentally there is no compelling evidence to favour the 
latter choice. Recently there has been a demise in propane use because of the extra handling precautions necessary and the safety difficulties of shipping it in Dewars.

Nitrogen is the cryogen of choice since it is cheap, abundant and safe, and in its liquid form always takes the crystal solvent below the critical phase transition at around $155 \mathrm{~K}$ (Weik, Kryger et al., 2001). Although other cryogens have been and

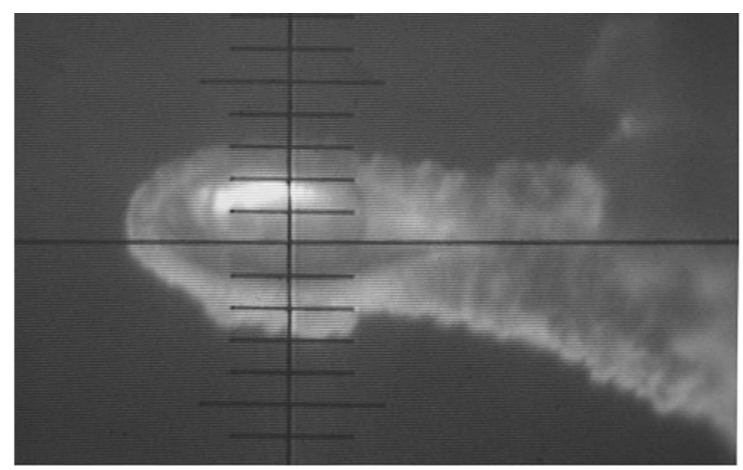

(a)

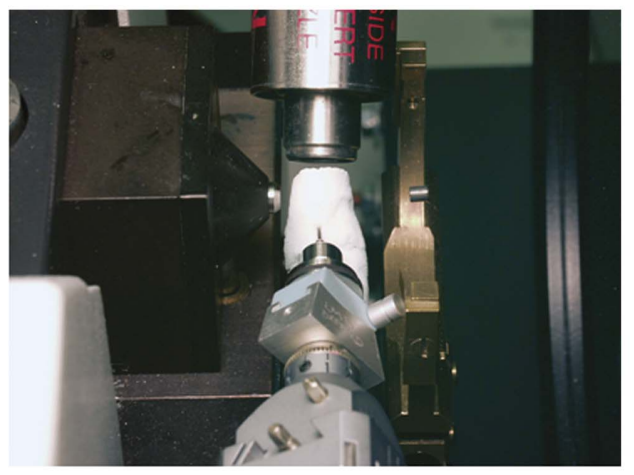

(b)

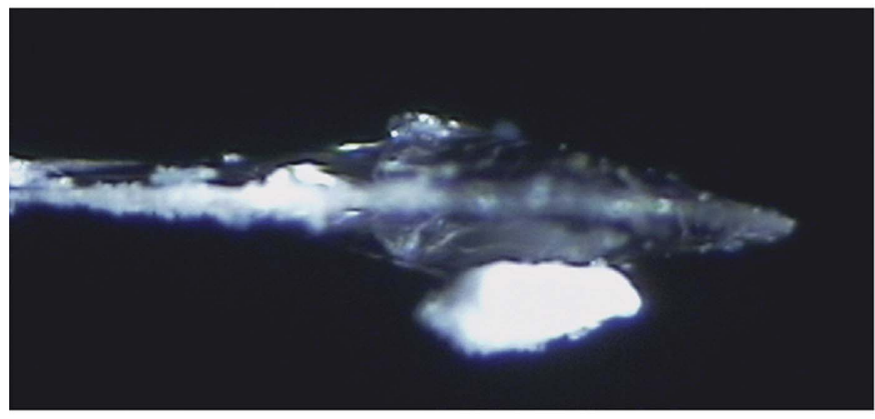

(c)

\section{Figure 5}

Examples of different icing problems. (a) Ice on, within and around sample. Loop too far from end of cryostat nozzle (5-8 $\mathrm{mm}$ recommended) and not correctly aligned in the cold nitrogen stream. After correction of alignment and distance, annealing could be tried and might rescue this sample. (b) Flow-rate of outside dry air/nitrogen stream not matched to inner cold nitrogen stream, so that turbulence on the gas interface draws in wet warm air from the room. Moisture from the air drops out giving a cylindrical wall of frost round the sample. Adjustment of the dry air/ nitrogen flow will clear this and for this sample, a data set was subsequently successfully collected. (c) An ice blob deposited on a crystal during storage or transport. This can be cleared by brushing the sample with a small artist's brush or pouring a small volume of liquid nitrogen over the loop (taking care not to crack the beamline camera lens if it is directly below the sample). are still being utilized, safety considerations and the problems of shipping flammable gases mean that in practice nitrogen is the preferred option. With nitrogen, there are two possibilities: plunge cooling into a small Dewar of liquid, or stream freezing using a goniometer and cryostat. Although for particular crystal species there is anecdotal evidence that one method is preferred over the other, there has so far been no systematic study that allows a rational decision to be taken, so it is usually a question of which is more convenient.

For stream-cooling there are some compelling reasons for blocking the gas stream with a piece of card or so called 'cryoshutter' while positioning the crystal on the goniometer, and then swiftly removing the card. This will: (i) avoid the crystal being dehydrated by dry nitrogen/air stream which surrounds the cryogen, (ii) avoid the crystal being waved in and out of stream while positioning it resulting in slow cooling which will give ice, and (iii) ensure that the crystal is cooled as fast as possible, so that vitrification is more likely.

A recent theoretical study modelling cooling rates for the flash-cooling of protein crystals in loops (Kriminski et al., 2003) concluded that the choice of cryogen was of relatively low importance to successful cryocooling. The crystal solvent content and solvent composition came top of the list, followed by the crystal size and shape (crystals with large surface to volume values cool faster and more uniformly than those with small $S / V$ ), amount of residual liquid around the crystal (which should be minimized), the cooling method (liquid or stream cooling), choice of gas/liquid cryogen and lastly the relative speed between the cooling agent and the crystal. This theoretical study has given some rationale to procedures which have been empirically determined over the last ten years.

Note that proper safety precautions such as the wearing of goggles and gloves (but preferably not sandals) should always be followed when using cryogens.

\subsection{Ice}

The formation of ice in, on and around the crystal is an irritation which can negatively affect the experimental outcome (see Fig. 5 for some examples). There is absolutely no need for ice to be a problem. For a full trouble-shooting guide see Garman \& Schneider (1997).

Common causes of ice on and around the crystal are: poor experimental geometry, misaligned cryostat nozzle (Fig. 5a), nozzle too far from the experiment, the outer sheath of dry air/ nitrogen flow rate not being matched to the cryogen flow rate (Fig. 5b), and a draughty environment. The stream of nitrogen must hit the crystal not the pin first, and prior to the experiment, a cryostat alignment nozzle (Mitchell \& Garman, 1994) can be used to ensure that the cryostat is properly centred. The end of the nozzle should ideally be $5-8 \mathrm{~mm}$ from the crystal. Shielding the crystal position from draughts helps ensure the crystal is kept at cryotemperatures the whole time.

Ice which accumulates on the crystal during data collection (Fig. 5c) can be removed using an acupuncture needle or an artist's brush. Alternatively a small volume of liquid nitrogen 
can be poured over it, but if the crystal viewing camera is directly below the crystal, beware of cracking the lens!

For a sufficiently protected pre-tested buffer, ice within the crystal and vitreous support is usually the result of slow cooling rather than flash-cooling, and can often be rectified by annealing. For an 'initial estimate' cryobuffer, it usually signifies that insufficient cryoprotectant agent was used.

\subsection{Annealing}

If the crystal has cooled poorly, it can be annealed (cycled between low and higher temperatures) to try to reduce the mosaicity and increase the resolution. Annealing can be carried out in two ways, either by blocking the gas stream temporarily (Yeh \& Hol, 1998) or by putting the crystal back in the cryoprotectant, allowing it to equilibrate and then flash cooling it again (Harp et al., 1998).

Annealing is sometimes spectacularly successful, but certainly not always. Thus, both methods of annealing outlined above are worth trying, unless there is only one crystal: in this scenario some data should be collected first in case annealing makes the diffraction worse. Kriminski et al. (2002) found that warming HEWL crystals up to between 230 and $250 \mathrm{~K}$ and then flash cooling them again gave more reproducible results than did warming up to $293 \mathrm{~K}$, and this may be a generally applicable strategy. From these experiments, understanding of the annealing process is now starting to emerge (Kriminski et al., 2002). In addition Juers \& Mathews (2004) have shown that bulk solvent leaves the crystal during annealing if the cryoprotectant agent concentration is below the optimum (defined as giving the best diffraction properties), and that water enters the crystal during annealing if the initial cryoprotectant concentration is higher than the optimum. The experimenters concluded that during annealing, the cryoprotectant agent concentration adjusts itself, thereby changing its thermal properties so that the bulk solvent contraction when cooled more nearly matches the contraction of the crystal lattice. They further found that annealing is more likely to be successful if the initial concentration of cryoprotectant agent is above, rather than below, the optimum. These results imply that it is better to err on the side of having too much, not too little cryoprotectant agent in the cryobuffer, as the crystal can then be annealed subsequently (Juers \& Mathews, 2004).

\subsection{Optimization}

If the diffraction is adequate for the purposes of the experiment, further optimization of the cryoprotocol is often unnecessary. However, if the diffraction is marginal for obtaining the required data, or if it is poor, optimization (after annealing has been tried) can profitably be carried out and Fig. 3 gives a flow diagram of a possible strategy for this process. It includes checking that the cryobuffer has been made optimally (i.e. not by dilution), modifying transfer and manipulation steps so that they are less harmful to the crystal (e.g. in situ serial transfer instead of moving the crystal each time the concentration is increased) and also testing the crystals for diffraction at room temperature, so that time is not wasted on crystals which diffract poorly even before they are subjected to cryocooling.

Room-temperature testing of crystals can conveniently be carried out by mounting the crystal in mother liquor in a loop and then covering it in a glass capillary sealed with Plasticene to the brim of the top-hat (Skrzypczak-Jankun et al., 1996). This is much easier than the traditional glass capillary mounting method, which has all but been abandoned in many laboratories but a procedure for which is presented in Garman (1999a).

Recent theoretical work (Halle, 2004) analysing the population of conformational states has raised questions about the biological relevance of structures determined at cryotemperatures, $150-200 \mathrm{~K}$ below the normal physiological range, especially in relation to strongly solvent coupled processes (e.g. weak ligand binding, conformational switching of solvent-exposed side chains and hydration states). Experimentally, this has been investigated in a high-resolution comparison of room-temperature and $100 \mathrm{~K}$ structures of PAK pilin, which indeed found that although there were only small differences to the structural core of the protein, the protein surface was more extensively affected (Dunlop et al., 2005). Thus, it seems advisable to collect a room-temperature data set if detailed biological mechanisms are being deduced from the $100 \mathrm{~K}$ structure.

A recent example from our home laboratory serves to illustrate the value of characterizing crystals at room temperature. First attempts at cryocooling crystals of a cyclin E1/CDK2 complex using a 'quick dip' in a cryobuffer of $30 \%(v / v)$ glycerol added to the mother liquor (which was thus diluted), resulted in diffraction to around $3.3 \AA$ with a mosaicity of $1^{\circ}$ (at beamline BM14, ESRF, Grenoble). The

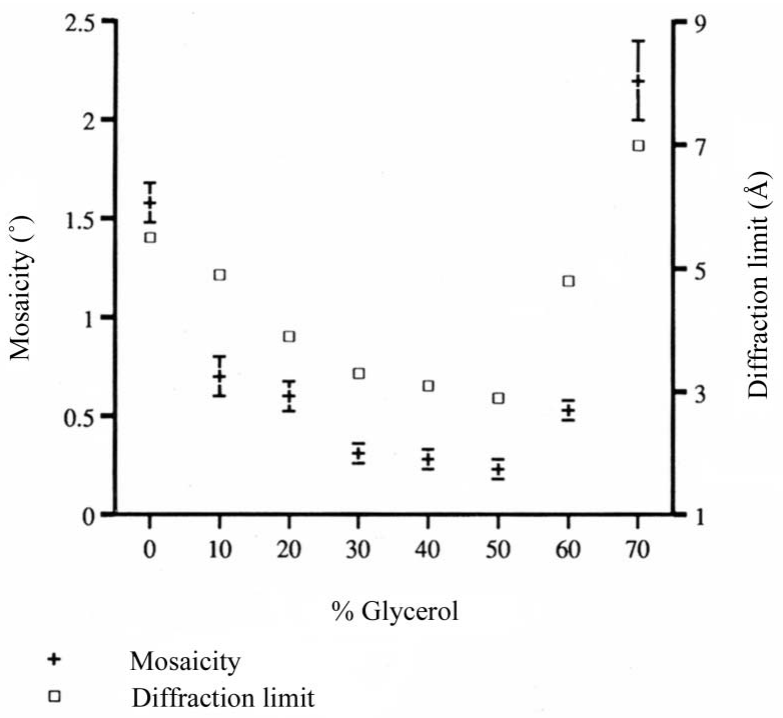

Figure 6

Graph showing the dependence of resolution limit and mosaicity on cryoprotectant agent concentration for crystals of rabbit muscle T-state glycogen phosphorylase $b$. The average values for measurements on three different crystals at each concentration are shown. There is a clear optimum between 40 and 50\% glycerol. Data from Mitchell \& Garman (1994). 
room-temperature mosaicity, measured on in-house rotating anode, was $0.5^{\circ}$ with diffraction to $3.4 \AA$. In-house testing of different cryoprotectant agents resulted in $25 \%(v / v)$ (made up by substituting the water in the mother liquor with cryoprotectant agent) ethylene glycol being used. A mosaic spread of $0.6^{\circ}$ and a resolution limit of $2.25 \AA$ (at beamline ID14-4, ESRF, Grenoble) was then obtained. The structure could then be solved by molecular replacement (Honda et al., 2005) and biological conclusions were drawn that would have been inaccessible had only a $3.3 \AA$ structure been obtained. This case gives a convincing argument for optimizing the cryoprotectant protocol if results are resolution limited!

Data quality is generally agreed to be best when the mosaicity is minimized, as the signal to noise ratio is higher and weak reflections can be measured more accurately. Thus, minimizing the mosaicity is one of the aims of optimization attempts. In theory it should be possible, with the right cryoprotocol, to reproduce the room-temperature mosaicity in cryocooled crystals, although to achieve this is often too difficult to be worth the effort.
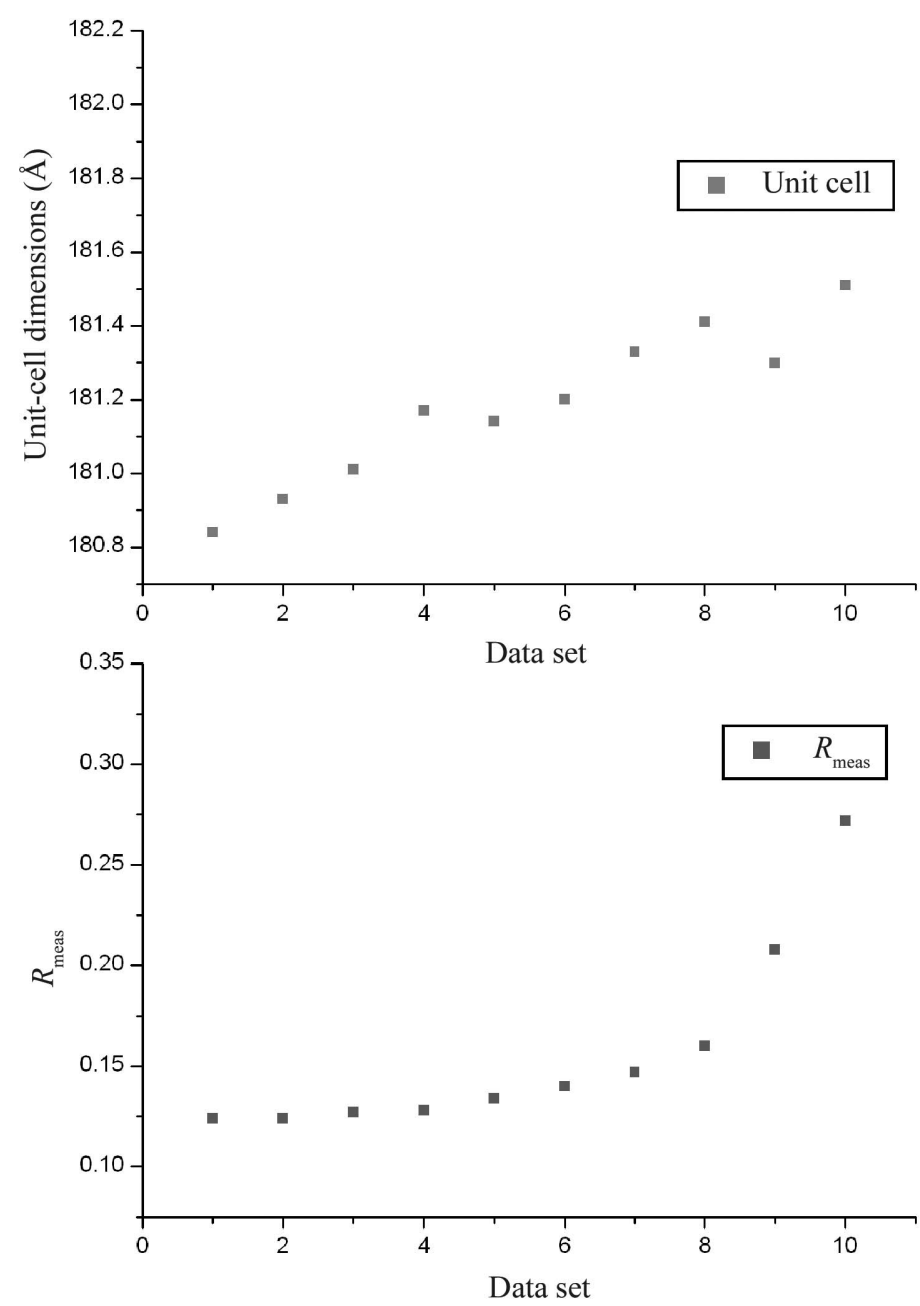

In practice it has been shown, for crystals of rabbit glycogen phosphorylase $b$, that there is an optimum cryobuffer concentration which both minimizes the mosaicity and maximizes the diffraction, and these results (Mitchell \& Garman, 1994) are shown in Fig. 6. Thus, systematic experiments to optimize the cryoprotocol for a particular crystal species can be rewarded when better data and more detailed biological information are obtained.

\subsection{Storage and transport}

Experimentally, the storage and retrieval of flash-cooled crystals is fraught with pitfalls. The success rate is lower than desirable, and ice is often a problem on retrieved crystals (see Fig. 5c). New hardware is currently being developed and distributed to accommodate crystal mounting robots, and thus success rates and ease of use should improve in the near future. The commercially available racking systems are more convenient than cryovials and hold temperature in a shipping Dewar very well (Owen et al., 2004).
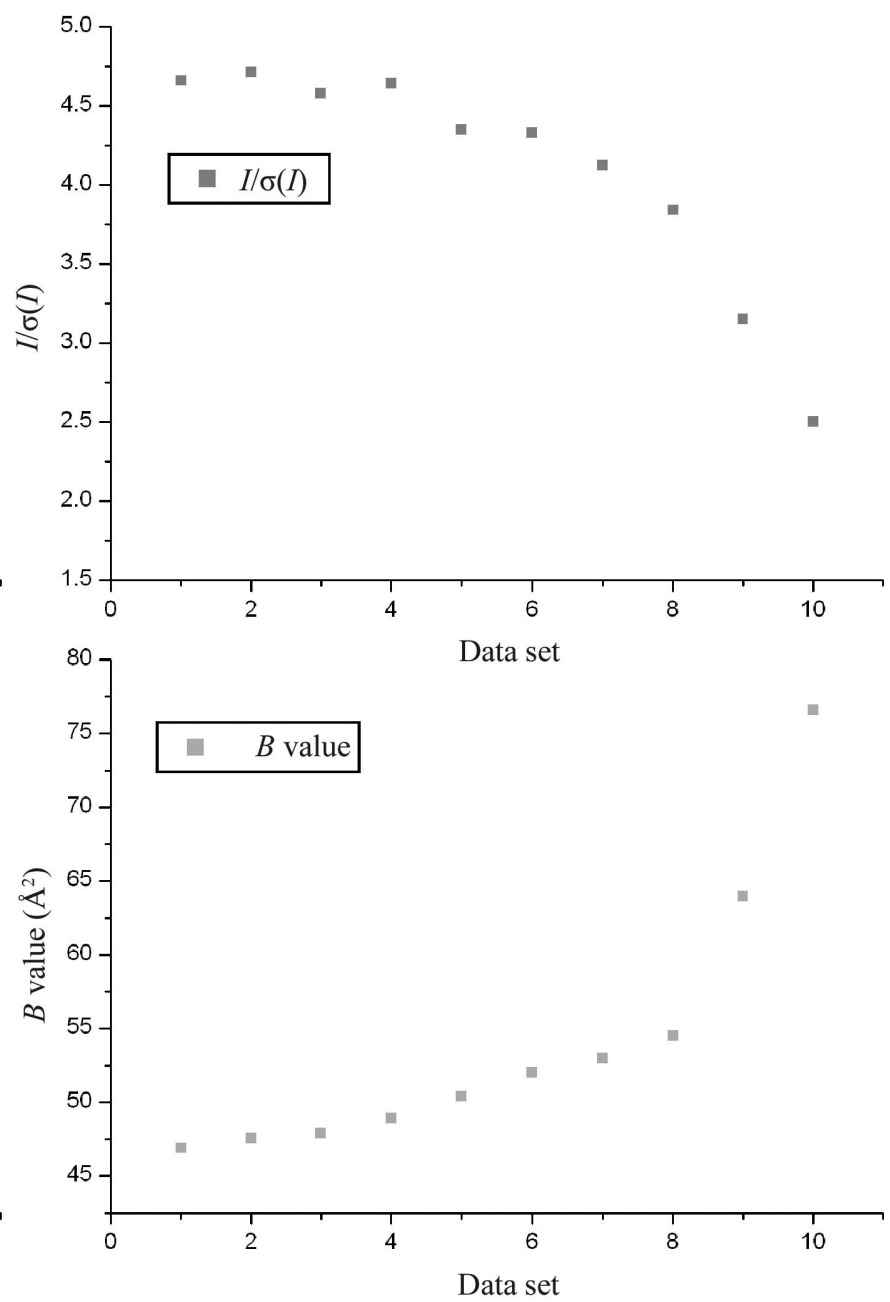

Figure 7

Effects of radiation damage on the characteristics of an apoferritin crystal exposed for ten complete data sets collected using an attenuated beam with a 'burn' by the unattenuated beam between each. Data were collected on ID14-4 at the ESRF, France. $(a)$ Unit cell $(F 432, a=181 \AA)(b) I / \sigma(I)(c) R_{\text {meas }}$ (d) Wilson $B$ value. 
Step-by-step instructions for the more common crystal manipulations using cryotongs and/or removable arcs can be found in Pflugrath (2004) and Garman \& Owen (2005), and at http://biop.ox.ac.uk/www/garman/gindex.html. The commercially available dry shipping Dewars really do hold low temperature as advertised if they are given some care (see the manufacturer's instructions). They should be properly dried out after every use, particularly if they were opened and closed many times at the synchrotron. Moisture can get into the absorption material and seriously compromise its cooling capacity: next time the Dewar is cooled with liquid nitrogen, the water freezes to ice in the material which then cannot absorb the nitrogen and thus cannot hold cryotemperatures.

\section{When cryocooling is not enough}

As already mentioned above, observation of radiation damage to cryocooled protein crystals has now become common at third-generation synchrotron sources. Radiation damage is inflicted by 'primary' interactions when the beam loses energy to the molecules in the crystal or solvent. This energy is dissipated in at least two ways: by providing the necessary energy to break bonds between the atoms in the molecules and also by the production of heat (thermal vibration of the

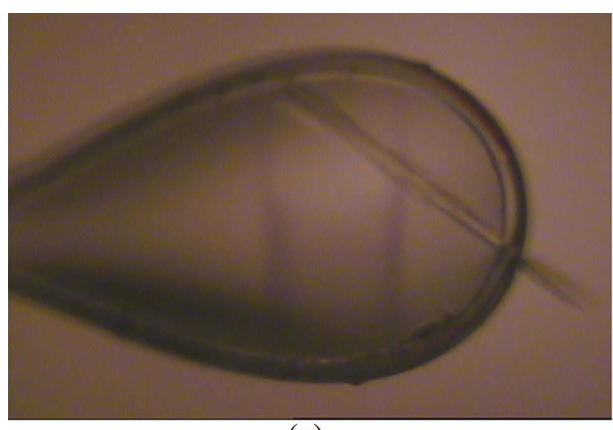

(a)

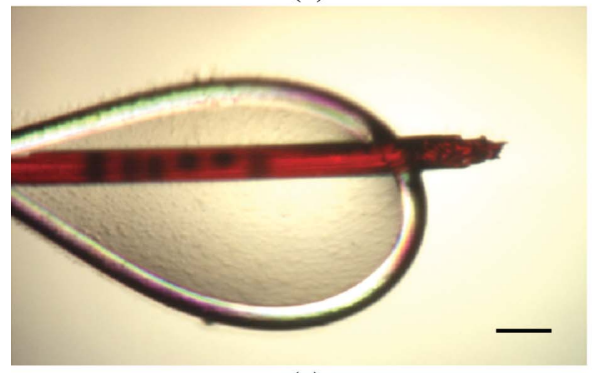

Figure 8

(c)

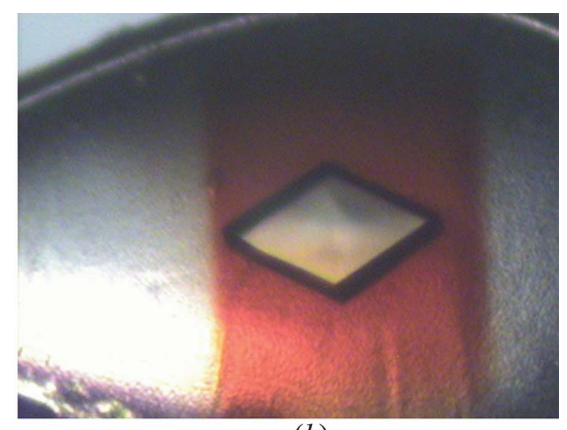

(b)

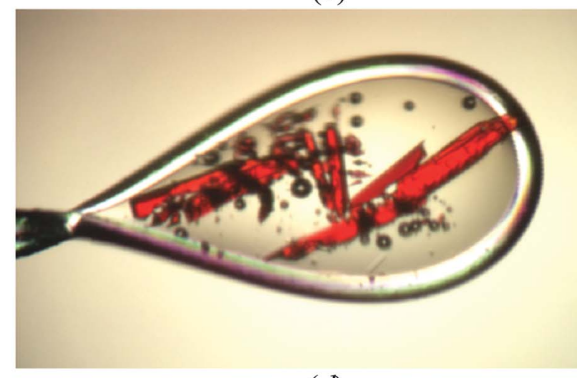

(d)
Visual changes to samples owing to X-ray irradiation. (a) A needle crystal (approx $20 \times 20 \times$ $300 \mu \mathrm{m}$ ) of complement component protein Factor I that has been exposed to a $100 \times 100 \mu \mathrm{m}^{2}$ beam at ID14-3, ESRF, Grenoble for a total of $45 \mathrm{~min}$. The cryobuffer was $25 \%$ PEG600 and $0.1 \mathrm{M}$ imidazole malate $\mathrm{pH}$ 4.5. (Roversi et al., 2004). (This photograph was kindly provided by Pietro Roversi.) (b) Crystal of Salmonella typhimurium neuraminidase cryocooled to $16 \mathrm{~K}$ using a helium gas stream at ID14-4, ESRF, Grenoble after a few seconds of X-ray exposure. The crystal was in phosphate buffer $\left(2 \mathrm{M} \mathrm{K}_{2} \mathrm{HPO}_{4}: 0.43 \mathrm{M} \mathrm{KH}_{2} \mathrm{PO}_{4}\right.$ ) with $40 \%$ glycerol as the cryoprotectant agent. The buffer alone is responsible for the colour change. (c) A crystal of bacteriorhodopsin exposed at $100 \mathrm{~K}$ for ten images at each of 13 places to a $30 \mu \mathrm{m}$ diameter X-ray beam on ID19 at the ESRF, Grenoble. $(d)$ After the exposures, the crystal was allowed to warm up to room temperature, and it disintegrated, releasing gas which can be seen as bubbles. The black scale bar represents $50 \mu \mathrm{m}$. (Photographs $c$ and $d$ were kindly provided by Tassos Perrakis.) molecules). The extent of this primary damage is dose ependent. The products can react and/or diffuse through the crystal causing further destruction ('secondary' damage). This compont of the radiation damage is time and temperature collection and is not reduced even if the sample is cooled. Since the mobility of radicals is much lower at $100 \mathrm{~K}$ than at room temperature, secondary damage is substantially reduced at cryotemperatues. However, we now know that it is not liminated (see below).

Radiation damage gives rise to changes in a number of generally observable parameters during and after the experiparticularly at high resolution, increase in unit-cell volume, increase in Wilson $B$ values, increase in $R_{\text {meas }}$, increase in the $B$ values of the refined structural model, and often (but not always) an increase in mosaicity. Visible changes in the samples are also observed (see Fig. 8). Of more direct relevance to the biological interpretation of structures, specific C-S bonds in methionines are cleaved (Burmeister, 2000; Ravelli \& McSweeney, 2000; Weik et al., 2000). Some examples oferritin crystal (which has no disulfide bonds) are shown in Fig. 9. Enzyme mechanisms can involve susceptible residues, so special care is required when interpreting structures which may have been modified by X-ray damage during the data collection. Metalloproteins are particularly susceptible to partial reduction during the diffraction experiment, and may not be in their native state by the end of the data collection (Carugo \& Djinovic Carugo, 2005).

In fact, researchers are becoming increasingly aware of the effects of radiation damage on the biological conclusions being drawn from structures. For example, in studies of the primary photoreaction of bacteriorhodopsin in conjunction with an online spectrophotometer (350-800 nm), a synchrotron X-ray beam induced half the protein to convert into an orange species during data collection, and further experiments at different radiation doses were necessary to identify the structural changes that are solely related to the light-cycle of bacteriorhodopsin (Matsui et al., 2002; Takeda et al., 2004). Active sites may be particularly vulnerable to damage: for instance, in the structure determination of DNA apophotolyase, the flavin 
adenine dinucleotide chromophore in the active site was reduced during X-ray data collection (Kort et al., 2004).

The specific structural damage combined with the gradual increase in unit-cell volume as the experiment proceeds induces non-isomorphism on two fronts. This gives problems with MAD structure solution, since by the time the third wavelength is collected, the cell and atomic structure can have changed such that the reflection intensities are significantly altered just by non-isomorphism. It has been calculated (Crick \& Magdoff, 1956) that an increase of $0.5 \AA$ in all unit-cell edges of a $100 \AA$ cubic cell changes the diffraction intensities by $15 \%$ at $3 \AA$ resolution. This value compares rather unfavourably with the $6-10 \%$ changes in intensity which must be

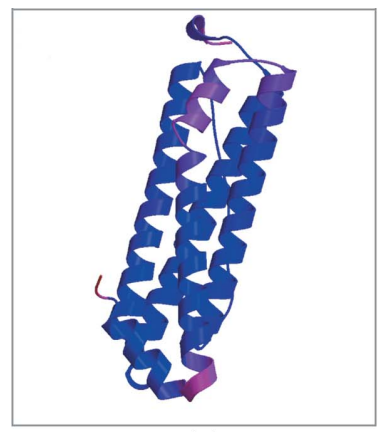

(a)

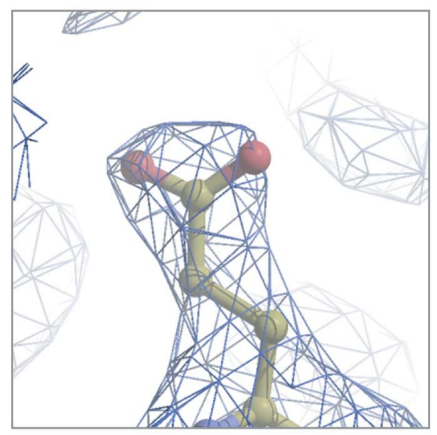

(c)

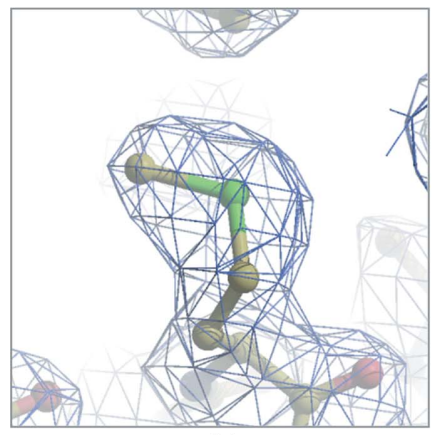

(e)

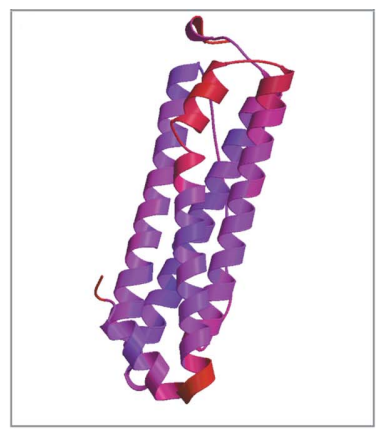

(b)

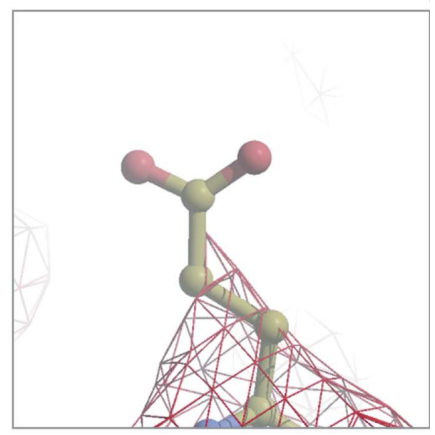

(d)

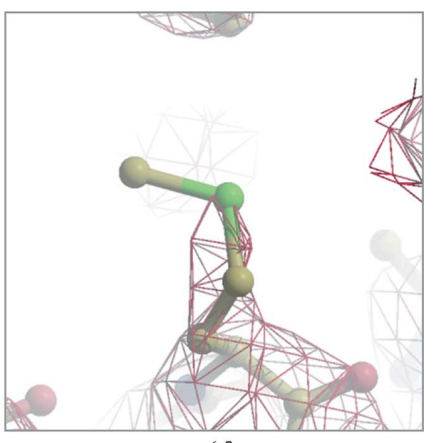

$(f)$

\section{Figure 9}

$B$-value increase and specific structural damage inflicted on a cryocooled crystal of apoferritin during sequential data sets collected at ID14-4, ESRF: $(a)$ and $(b)$ structure coloured from blue $\left(20 \AA^{2}\right)$ to red $\left(100 \AA^{2}\right)$ by crystallographic $B$-value for $(a)$ data set 1 and $(b)$ data set 10 [plotted using Molscript (Kraulis, 1991) and Raster3d (Merritt \& Bacon, 1997)], (c) $2 F_{\mathrm{o}}-F_{\mathrm{c}}$ map of Glu63 contoured at $0.2 \mathrm{e} \AA^{-3}$ after data set 1 and $(d)$ after data set 10 . (e) $2 F_{\mathrm{o}}-F_{\mathrm{c}}$ map of Met 96 contoured at $0.2 \mathrm{e} \AA^{-3}$ after data set 1 and $(f)$ after data set 10, showing specific structural damage [maps are from Coot (Emsley \& Cowtan, 2004), and plotted using Raster3d]. detected to solve the phase problem in a typical MAD experiment (Hendrickson \& Ogata, 1997).

Thus, because of the negative influence of radiation damage on the success rate of MAD structure solution, and the effect it has on the interpretation of biological results, radiation damage at cryotemperatures has now become an issue of wide concern to the structural biology community.

\section{Mechanisms of radiation damage}

For a beam wavelength of $1 \AA$ (12.4 keV), approximately $98 \%$ of the X-rays incident on a typical protein crystal pass straight through it without interacting, and are absorbed by the beamstop. The remaining $2 \%$ may interact in one of three ways; by the photoelectric effect $(84 \%)$, by Compton scattering $(8 \%)$ or by Thomson (Rayleigh) scattering $(8 \%)$. Thomson scattering is elastic and coherent, and by vectorial addition of the components it results in the observed diffraction pattern. Compton scattering is inelastic and incoherent, and adds to the background in the images. Most of the energy that is deposited in the crystal is via the photoelectric effect, in which the energy of the photon is used to eject a lower shell electron from the atom: the electron will carry the energy of the incoming photon minus the ionization energy. This photoelectron, which can be formed in either the solvent ('indirect' effect) or the protein ('direct' effect), then moves through the crystal losing energy in up to 500 scattering events giving rise to the formation of radicals (O'Neill et al., 2002), including $\mathrm{OH}, \mathrm{H}, \mathrm{H}^{+}$and hydrated electrons $\left(\mathrm{e}_{\mathrm{aq}}^{-}\right)$from the radiolysis of water. The mobility of most of these radicals is greatly reduced at cryogenic temperatures resulting in the extended lifetimes of cryocooled crystals.

Further damage occurs when the radicals (particularly $\mathrm{e}_{\mathrm{aq}}^{-}$) interact with the protein. The stability of the carbonyl radical means that it is possible that the carbonyl group of the peptide bond acts as a major trapping centre for electrons. Excess electrons can then migrate along the peptide chain via the hydrogen bonds between peptide units (reviewed by Garrison, 1987). Susceptible side chains can then become the loci for specific structural damage.

It is interesting to note that if the specific damage were a result of primary damage alone, it would occur in the order of absorption cross sections of atoms, the biggest showing damage first. In fact, there is a somewhat better correlation between the covalent binding energies of the interatomic bonds (weakest being damaged first) in a protein (see Table 2) than between the absorption cross section and the order of damage observed in a cryocooled protein crystal. Thus, the specific damage must involve secondary effects. Electrons are known to be mobile in proteins held at $77 \mathrm{~K}$ from electron spin resonance (ESR) measurements (Jones et al., 1987), and could thus 'channel' the energy deposited in the crystal to weaker bonds.

Little can be done to prevent primary damage, though careful choice of the wavelength of radiation used can have a significant effect on the amount of energy absorbed by a crystal if it contains a heavier scatterer (above or below its 
Table 2

Covalent bond strengths of linkages common in proteins.

The $\mathrm{C}-\mathrm{S}$ bond is the weakest, consistent with the observation that the $\mathrm{S}$ atoms in disulfide bonds are the first to delocalize when protein crystals at $100 \mathrm{~K}$ are irradiated. Note that the polypeptide main chain is stabilized by geometry and that bond strengths are highly dependent on the local environment, which can cause bonds to be strengthened or weakened.

\begin{tabular}{lll}
\hline Bond & \multicolumn{2}{c}{ Bond strength } \\
\cline { 2 - 3 } & $\mathrm{kJ} \mathrm{mol}^{-1}$ & $\mathrm{eV}$ \\
\hline $\mathrm{C}-\mathrm{S}$ & 260 & 2.7 \\
$\mathrm{C}-\mathrm{N}$ & 293 & 3.0 \\
$\mathrm{~S}-\mathrm{H}$ & 340 & 3.5 \\
$\mathrm{C}-\mathrm{C}$ & 349 & 3.6 \\
$\mathrm{C}-\mathrm{O}$ & 358 & 3.7 \\
$\mathrm{~N}-\mathrm{H}$ & 391 & 4.1 \\
$\mathrm{~S}-\mathrm{S}$ & 412 & 4.3 \\
$\mathrm{C}-\mathrm{H}$ & 415 & 4.3 \\
$\mathrm{O}-\mathrm{H}$ & 463 & 4.8 \\
$\mathrm{C}=\mathrm{O}$ & 740 & 7.7 \\
\hline
\end{tabular}

absorption edge). The heavier an atom, the larger its photoelectric cross section (the atomic absorption coefficients increase in approximate proportion to the fourth power of the atomic number), hence a heavy-atom derivative will absorb a larger dose than a native crystal (Murray et al., 2005): in fact the addition of one $\mathrm{Hg}$ atom per protein molecule in a crystal of lysozyme will double the absorption (i.e. $4 \%$ of a $1 \AA$ incident beam will interact as opposed to $2 \%$ for a native crystal).

\section{Absorbed dose}

The dose absorbed by a sample when irradiated is measured in units of Gray, and is defined as the energy deposited per kilogram of sample material. In an MX (macromolecular crystallography) experiment, the absorbed dose is a function of both the beam parameters (size, shape, flux and energy) and the crystal composition. The latter can be determined via knowledge of the protein sequence and the solvent constituents, allowing the absorption coefficients for an X-ray beam of a particular energy to then be computed. It can be problematic to find out the characteristics of the beam at a synchrotron beamline: particularly the incident flux, which must be determined for each experiment using a calibrated pin diode. However, knowledge of the flux is essential if a reasonable estimate of absorbed dose is to be made. This is thus an issue that will become more important as MX techniques are increasingly used on weakly diffracting crystals of large protein complexes that require the full flux of undulator beamlines to obtain measurable diffraction.

A theoretical calculation of the dose limit for half the diffraction intensity of a protein crystal to be lost has been made (Henderson, 1990) as $2 \times 10^{7}$ Gy by analogy with the dose observed to destroy half of the electron diffraction in samples examined at $77 \mathrm{~K}$ by electron microscopy. This dose is delivered by around five $100 \mathrm{keV} \mathrm{e} \AA^{-2}$ (Chiu et al., 1986). From the energy loss of this electron flux density and the mean range of penetration of electrons in a protein embedded in glucose, an absorbed dose of $5 \times 10^{7} \mathrm{~Gy}$ is obtained. The limit of $2 \times 10^{7}$ Gy results from assuming that the dose absorbed in the first part of the depth-dose curve is two to three times less.

This theoretical limiting dose of $2 \times 10^{7} \mathrm{~Gy}\left(\mathrm{~J} \mathrm{~kg}^{-1}\right)$ can be converted into the energy loss in a typical protein crystal, and thus used to calculate the number of $8 \mathrm{keV} \mathrm{X-ray} \mathrm{photons}$ required to deliver it $\left(12 \times 10^{-16} \mathrm{~Gy}\right.$ photon ${ }^{-1} \mathrm{~m}^{-2}$ so a total of $1.6 \times 10^{16}$ photons $\mathrm{mm}^{-2}$ ), assuming that electrons and $\mathrm{X}$-rays are similarly damaging to protein structure at cryotemperatures. Since an in-house rotating anode generator with confocal optics delivers around $2.5 \times 10^{8} \mathrm{keV}_{\text {photons s}}{ }^{-1}$ into approximately a $300 \times 300 \mu \mathrm{m}$ spot, the theoretical dose limit would be reached in about 10 weeks, whereas a microfocus rotating anode can give $\sim 7 \times 10^{8} \mathrm{keV}$ photons $\mathrm{s}^{-1}$ into a $150 \times 150 \mu \mathrm{m}$ spot, giving only $6 \mathrm{~d}$ to reach a dose of $2 \times 10^{7} \mathrm{~Gy}$. However, on a third generator unattenuated undulator beamline (flux density of around $10^{12}$ photons per $100 \times 100 \mu \mathrm{m})$ this would only take about $10 \mathrm{~min}$ total exposure for a $13.2 \mathrm{keV}$ beam.

It is important to note that since the theoretical dose limit takes into account only the physics of the energy loss in the crystal, not its chemistry, the limit gives an idea of the maximum length of time a crystal might last, rather than a hard value. For instance, if a crystal has particularly radiation susceptible amino acids (e.g. glutamates) forming the only crystal contacts, radiation damage may cause the crystal to lose its order long before predicted by the Henderson limit [see for example dodecin (Murray et al., 2005)].

Dose calculations are made easier by the use of programs such as RADDOSE (Murray et al., 2004), which given the beam parameters and crystal composition will calculate the absorbed dose. RADDOSE also gives a total exposure time to reach $2 \times 10^{7} \mathrm{~Gy}$, allowing the experimenter to plan an experiment with some idea of the likely maximum lifetime of the crystal.

The anomalous scattering power of an atom is directly proportional to its photoelectric cross section, so the more favourable an atom/wavelength combination is for phasing, the greater the absorbed dose. Thus, at the optimum wavelength for SAD data collection or peak wavelength for MAD, absorption is maximized and thus the rate of radiation damage is also highest. As isomorphous and complete data are highly desirable for anomalous phasing, a careful strategy is required for successful data collection.

\section{Investigating and reducing radiation damage}

The rate of X-ray radiation damage in a crystalline biological sample is thought to depend on a large number of parameters including those governing the external variables of the experiment: the beam flux, flux density, energy, size and profile, and the cryocooling regime, and those influencing the physical and chemical properties of the crystal. These are shown schematically in Fig. 10. The effects of changing these parameters are being actively investigated; understanding of some is further advanced than for others. This on-going research is now too extensive to be reviewed in detail here and 
the reader is referred to the references given below for further information.

There is currently no foolproof way to monitor damage online, both because crystals of the same protein species show significant variability in behaviour $\left[R_{\text {meas }}\right.$, unit-cell volume increase mosaicity, and $I / \sigma(I)]$ and also since specific structural damage is known to occur before the diffraction pattern shows visible degradation (Ravelli \& McSweeney, 2000). Thus, mitigation strategies are hard to test quantitatively other than by looking at the rate of specific structural damage, which is both time consuming and labour intensive. Unfortunately, unit-cell expansion, although linear with dose, has been shown to be irreproducible in rate among different crystals of the same protein (Murray \& Garman, 2002; Ravelli et al., 2002). Thus, the best way to monitor the damage is to know the absorbed dose for the data collection.

\subsection{External variables}

6.1.1. Cryogen temperature during data collection. In current practice, a gaseous nitrogen stream held at 90-110 K cools the crystal during data collection. Open flow helium is an alternative cryogen and can reach lower temperatures (down to $\sim 16 \mathrm{~K}$ ). Theoretically, high-resolution diffraction should be enhanced at lower temperatures due to reduced $B$ values, although this effect is only likely to be significant for data better than $1.5 \AA$ (Garman, 1999b). It appears from some experimental studies that higher resolution, improvement of data quality and extended lifetime may sometimes be obtained (Teng \& Moffat, 2002; Hanson et al., 2003): signal-to-noise ratios can certainly improve. However, there have been a number of unpublished experiments carried out at different synchrotrons, including several by the authors (at SSRL, USA and ESRF, France) where no clear improvement or advantage was identified. There are serious cost implications if helium

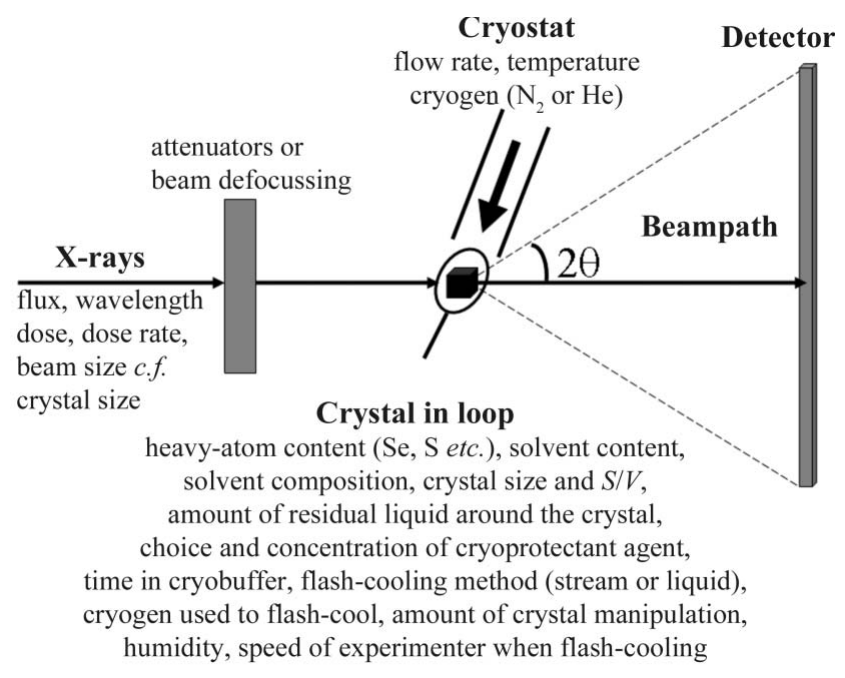

Figure 10

Schematic representation of the diffraction experiment, annotated to show the parameters believed to be relevant to radiation damage progression and cryocooling outcomes. were to be adopted as the preferred cryogen at synchrotrons, and so far the experimental evidence is certainly not persuasive. Helium has a larger heat-transfer coefficient than nitrogen and so even at $100 \mathrm{~K}$, vitrification during flashcooling is faster (Kriminski et al., 2003). Thus, less cryoprotectant agent is required, and for cases where no satisfactory cryoprotocol can be found, helium is thus worth trying if it is available.

6.1.2. Heating of the crystal by the beam. One of the ways that the energy of the synchrotron X-ray beam is dissipated in a crystal is by heat production, resulting in a rise in crystal temperature. The magnitude of this effect has been calculated by finite element analysis (Nicholson et al., 2001) and by analytical methods (Kuzay et al., 2001). The latter study concluded that for a $100 \mathrm{~K}$ nitrogen cooling regime and a $100 \mu \mathrm{m}$ thick crystal, the sample temperature rise for a flux of $10^{13} \mathrm{ph} \mathrm{s}^{-1} \mathrm{~mm}^{-2}$ would be about $6 \mathrm{~K}$ at equilibrium. In a further detailed theoretical analysis (Kriminski et al., 2003), these results were confirmed for small crystals, but for larger $(200 \mu \mathrm{m})$ crystals held in nitrogen at $100 \mathrm{~K}$ in a flux density of $\sim 3 \times 10^{14} \mathrm{ph} \mathrm{s}^{-1} \mathrm{~mm}^{-2}$, heating of $20 \mathrm{~K}$ was predicted. Experimentally, no evidence of a significant temperature rise was observed up to $4 \times 10^{12} \mathrm{ph} \mathrm{s}^{-1} \mathrm{~mm}^{-2}$ by lattice expansion measurements on an organic light-atom crystal (Müller et al., 2002). Recent use of more advanced computational fluid dynamics techniques (Mhaisekar et al., 2005) predicted a $7 \mathrm{~K}$ rise in a $200 \mu \mathrm{m}$ diameter crystal in a beam of $4 \times$ $10^{14} \mathrm{ph} \mathrm{s}^{-1} \mathrm{~mm}^{-2}$ into $100 \mu \mathrm{m}$ slits being cooled by nitrogen at $100 \mathrm{~K}$. Thus, unless the crystal contains many heavy atoms which absorb a high dose per incident photon [e.g. holoferritin (Ravelli et al., 2002)], heating of the crystal is not likely to be a major factor in the rate of radiation damage for current undulator photon fluxes.

If the crystal heats to above $155 \mathrm{~K}$, the protein local conformational flexibility increases and the rate of radiation damage drastically accelerates (Weik, Ravelli et al., 2001).

Reliable experimental measurements of the heat rise in a protein crystal are notoriously hard to make because the measuring device carries heat into the system though its cables. An infrared camera has been used to thermal image both the cryocooling and annealing process (Snell et al., 2002). While these measurements are so far qualitative, when calibrated this technique promises to be a useful new tool for fundamental radiation damage and cryocrystallography research.

6.1.3. Beam energy. Although anecdotally shorter wavelength (higher energy) X-rays have been said to be less damaging than longer wavelength radiation, this assertion does not stand up to detailed scrutiny when the experiment is taken as a whole. Longer wavelength X-rays are more strongly absorbed by the crystal, but they are also more strongly diffracted (Arndt, 1984). In fact the diffracted intensity per absorbed dose ['diffraction-dose efficiency' (Murray et al., 2004)] changes only very slowly with incident energy for native, non metal-containing proteins. There are several additional experimental difficulties with collecting data at longer wavelengths; the detector response may be wavelength 
dependent, the Bragg angles are large for higher resolution reflections, and there will be more absorption of diffracted photons by the air between the crystal and the detector, although this can be minimized by use of a helium path. At very short wavelengths there are also experimental challenges: detector sensitivity, low-resolution reflections are behind the beamstop, high flux is required, and the mirrors must be especially designed.

A recent systematic comparison of the radiation damage inflicted by 1 and $2 \AA$ X-rays on crystals of porcine pancreatic elastase concluded that there were no significant qualitative or quantitative differences in damage (Weiss et al., 2005).

The energy of the beam relative to the absorption edges of any heavy atoms present will pivotally affect the dose absorbed by the crystal (much higher above the edge: see $\S 6.2 .1$ below).

6.1.4. Dose/dose rate effects. An important question pertinent to practical data-collection strategies is whether or not there is a dose/dose rate effect: would it be better to attenuate the beam by a factor of ten and expose the crystal for ten times longer to obtain the same counting statistics? Experiments to characterize the relationship between damage progression and dose have shown it to be linear up to $1 \times$ $10^{7} \mathrm{~Gy}$ (Teng \& Moffat, 2000). This upper dose limit seems to hold between 40 and $150 \mathrm{~K}$ (Teng \& Moffat, 2002). However, specific structural damage has been investigated as a function of dose/dose rate, and found to be greater at higher dose rate (Leiros et al., 2001). A different study that searched for a dose/ dose rate effect by analysis of data-reduction statistics, indicated that damage depends only on absorbed dose and found no evidence for any dose rate effect up to $10^{15} \mathrm{ph} \mathrm{s}^{-1} \mathrm{~mm}^{-2}$ (Sliz et al., 2003). However, for holoferritin, which has a highly absorbing iron core containing around $2000 \mathrm{Fe}$ atoms, a doserate effect has been reported at lower fluxes, attributed to a calculated crystal temperature rise of $\sim 100 \mathrm{~K}$ (Ravelli et al., 2002). Further experiments are currently in progress, and their results will affect future optimization of data-collection strategies.

\subsection{Physical and chemical properties of the crystal}

6.2.1. Crystal content. The presence of heavier elements in the crystal has a disproportionate effect on the absorbed dose since, as mentioned above, the atomic absorption coefficients increase in approximate proportion to the fourth power of the atomic number. The dose will be increased by a heavy atom soaked into the crystal to perform an SIR/MIR (e.g. Hg, Pt, Au or $\mathrm{Pb}$ ) or SAD/MAD (Se-Met) experiment and thus the crystal lifetime will be diminished. Similarly, any endogenous heavy atoms will increase the absorbed dose (e.g. $\mathrm{Cu}, \mathrm{Fe})$. Thus, for instance, a selenomethionine crystal will be expected to suffer faster damage than a crystal of the native protein (Murray et al., 2005).

To minimize this effect, crystals soaked in heavy-atom compounds can be back soaked to remove disordered absorbers, and thought can be given to exchanging heavy components in the mother liquor. The wavelength at which data are collected also affects the absorbed dose (the crystal will suffer increased dose above an absorption edge), and using $R A D D O S E$, experimenters can calculate the magnitude of this effect (Murray et al., 2004).

6.2.2. Minimum crystal size. The issue of how radiation damage affects the minimum useful crystal size for MX has been addressed in calculations by Gonzalez \& Nave (1994); Neutze et al. (2000); Glaeser et al. (2000); Teng \& Moffat (2000) and Sliz et al. (2003). They all arrived at different minimum crystal sizes dependent on the protein in question and the criteria set for useful data. The issue of minimum crystal size is very important in view of current developments towards high-throughput crystallography at an increasing number of facilities. Resolving these issues requires better definition of 'useful data' versus dose absorbed and systematic experimental data which would test the various assumptions made in the calculations.

A recent theoretical analysis has suggested that if crystals of less than $5 \mu \mathrm{m}$ in size are used, a significant number of the photoelectrons produced by the incident beam would escape from the crystal, thereby reducing the energy deposited and thus the radiation damage rate (Nave \& Hill, 2005). The magnitude of this effect increases with the energy of the incident photons, and is significant at $30 \mathrm{keV}$.

6.2.3. Addition of radical scavengers. Since the existence of specific structural damage at $100 \mathrm{~K}$ shows that some radicals are still mobile in the crystal (see above), free-radical scavengers might be able to react with these species and reduce their mobility and reactivity to prevent them interacting with the protein. Scavengers are used extensively and successfully in other branches of science. For protein crystallography, the beneficial effects of the scavenger must outweigh the extra dose caused by increased absorption when it is added to the crystal. Thus, for instance, metal containing scavengers such as $\mathrm{FeCl}_{3}$ significantly increase the absorbed dose (as calculated using RADDOSE), and would have to be very efficacious to result in an overall improvement.

We have investigated the use of some scavengers experimentally, and shown that styrene [used in the past in room-temperature MX (Zaloga \& Sarma, 1974)] is not effective. However, analysis of electron-density maps, coupled with monitoring the formation of a disulfide-related radical species at $400 \mathrm{~nm}$ with an off-line microspectrophotometer, showed that co-crystallization with $0.5 \mathrm{M}$ sodium ascorbate protected lysozyme (Murray \& Garman, 2002). Soaking of ascorbate into crystals of $\mathrm{N} 9$ neuraminidase from influenza virus cryoprotected with glycerol has also been found to be effective, although glucose (with glycerol) gave no extra benefits (Betts, 2003). Some cryoprotectant agents have potential roles as scavengers or conversely may accelerate the rate of radiation damage (O'Neill et al., 2002). Thus, any conclusions from these experiments may be dependent on the particular cryoprotectant agent used as well as the scavenger.

From our studies to date, we think that the use of radical scavengers is unlikely to give a decrease of more than a factor of 3 or 4 in the rate of radiation damage. 


\subsection{Correcting data for radiation damage}

For room-temperature data collection on a diffractometer with a one-dimensional detector, it was common practice to monitor several reflection intensities over the course of the experiment and to use a new crystal when the reference reflections had dropped to $70 \%$ of their time zero strength. Blake and Phillips (Blake \& Phillips, 1962) corrected their reflection intensities for radiation decay and so observed that the intensities could both increase as well as decrease with time.

Software correction procedures for extrapolating individual reflection intensities back to their zero dose level (given multiple measurements of each reflection) are being developed (Diederichs et al., 2003) and have been found to be effective for improving the phasing power of both longwavelength sulfur SAD data (Weiss et al., 2004) and selenium SAD data (Ravelli et al., 2005). Different (polynomial) parameterizations of this correction which best model experimental behaviour have been investigated for thaumatin (Banumathi et al., 2004). In another development, the program SHARP (de La Fortelle \& Bricogne, 1997) has been modified to allow the occupancy of heavy-atom sites to change during data collection: reflections remain unmerged and have a 'dose-stamp' associated with them. This strategy was tested and found to be successful on long-wavelength triiodide SAD data (Evans et al., 2003) and on a MAD structure solution which had previously failed because of radiation-damageinduced debromination of the RNA in the crystal during the experiment (Ennifar et al., 2002), but which could be solved by SAD following dose-related occupancy refinement of the bromine sites (Schiltz et al., 2004).

These software advances should enable the useful lifetime of the crystal to be extended and these ideas are already being incorporated into the standard data-processing software [e.g. zero-dose extrapolation is now in XSCALE (Kabsch, 1988)].

\section{Utilizing radiation damage}

The potential for using radiation damage effects to advantage is also being investigated, and is leading to some exciting new experiments both biologically and in the area of techniques development.

For instance, radiation damage has been used, in conjunction with more conventional data collection and off-line microspectrophotometer monitoring, to eludicate the catalytic pathway of horseradish peroxidase. The catalytic reduction of a bound dioxygen species was induced by electrons liberated during X-ray irradiation (i.e. by 'radiation damage') and was tracked using an ingenious multicrystal experimental strategy. Data sets comprising $90^{\circ}$ in $\varphi$ were collected from nine different crystals with the starting $\varphi$ staggered by $10^{\circ}$ for each. Composite $90^{\circ}$ data sets were assembled by taking the $10^{\circ}$ sweep from each crystal which had experienced the same $\mathrm{X}$-ray dose. Electron-density maps calculated from each composite data set gave 'snapshots' of the enzyme in various stages of reduction, similar to a redox titration. The radiation- damage process could potentially be utilized to investigate high valency intermediates of other redox enzymes. Also, unnoticed it may have affected the oxidation state of many redox proteins in the PDB (Berglund et al., 2002).

On the methods development front, a new way of phasing macromolecular structures has been established using the specific structural damage inflicted by X-rays. In the RIP (radiation-damage-induced phasing) method, a low-dose (with attenuators) data set is collected, followed by a 'burn' (no beam attenuation) of approximately half the Henderson dose limit. This dose destroys the disulfide bonds and causes other specific damage. A second low-dose data set is then collected, and the phases obtained from finding the 'heavy' (i.e. sulfur) atom sites. The principle has been demonstrated for a protein with six disulfide bridges; bovine trypsin, and for a DNA/RNA hybrid with brominated guanine, where radiation damage caused debromination (Ravelli et al., 2003). Although using RIP alone for structure solution may be confined to a limited number of favourable cases, when combined with, for example, sulfur SAD, it can make the difference between success and failure [e.g. Weiss et al. (2004) and also a recent structure solution of the ${ }^{2} \mathrm{~F} 1^{3} \mathrm{~F} 1$ modules of human fibronectin, for which experimental RIP and S-SAD phases were required in combination]. In fact, RIPAS (radiation-damage-induced phasing with anomalous scattering) has been shown to be an effective strategy for locating sub-structure and then phasing crystals of iodinated thaumatin (Zwart et al., 2004). The extra phasing information obtained by dose-dependent heavy-atom occupancy modelling in SHARP (see above) can also be classified as RIPAS.

\section{Conclusions}

Advances in cryocrystallographic techniques for macromolecular crystallography have been intimately intertwined with efforts to reduce the deleterious effects of X-ray damage inflicted during the collection of diffraction data. Cryoprotocols are now well established, but the approach is often haphazard: time invested in optimizing conditions can be very worthwhile in terms of improved diffraction leading eventually to more detailed biological information.

During the last five years it has become apparent that the age-old problem of radiation damage has not disappeared, although it is hugely reduced by cooling the sample to cryotemperatures. Concerted efforts to understand the relevant physico-chemical effects in vitreous samples held at $100 \mathrm{~K}$ are under way. There is now a more 'non-anecdotal' approach to the investigations, but statistically significant samples are hard to obtain and labour intensive to process. Some progress is being made, and the research has prompted some exciting new approaches such as RIP/RIPAS, and 'time-resolved in situ reduction' cryocrystallography.

For generally applicable experimental mitigation strategies to be effective, it will in the future be vital to be able to calculate the absorbed dose for a particular crystal, and for this knowledge of the incident flux as well as the beam size, profile and crystal size will be a requirement. 
As far as the practicing structural biologist is concerned, a knowledge of the artefacts that can be introduced by radiation damage is important when interpreting structures.

The understanding, mitigation and use of radiation damage at cryotemperatures has emerged very recently as a new and important area. Eventually, we might yet be able to fully utilize the flux of the undulator beamlines at cryotemperatures, overcoming the crystallographer's current dilemma: rate of damage versus diffraction intensity.

We are grateful for discussions with many colleagues on the issues covered in this paper and we would particularly like to acknowledge Sean McSweeney, James Murray, Colin Nave, Raimond Ravelli, and Martin Weik for stimulating exchanges. We thank Raimond Ravelli for his helpful comments on this manuscript. The ESRF, Grenoble has greatly supported our research efforts over the last five years with the award of a BAG for Radiation Damage studies. RLO is funded by a BBSRC CASE (Oxford Cryosystems) studentship. The program $R A D D O S E$ can be obtained from EFG.

\section{References}

Arndt, U. (1984). J Appl. Cryst. 17, 118-119.

Banumathi, S., Zwart, P., Ragagopal, U. A., Dauter, M. \& Dauter, Z. (2004). Acta Cryst. D60, 1085-1093.

Berglund, G. I., Carlsson, G. H., Smith, A. T., Szöke, H., Hendriksen, A. \& Hadju, J. (2002). Nature (London), 417, 463-468.

Betts, S. (2003). Part II thesis, Oxford University, England. (Unpublished work.)

Blake, C. C. F. \& Phillips, D. C. (1962). Biological Effects of Ionizing Radiation at the Molecular Level, pp. 183-191. Vienna: International Atomic Energy Agency.

Burmeister, W. P. (2000). Acta Cryst. D56, 328-341.

Carugo, O. \& Djinovic Carugo, K. (2005). Trends Biochem. Sci. 30, 213-219.

Chiu, W., Downing, K. H., Dubochet, J., Glaeser, R., Heide, R. M., Knapek, E., Kopf, D. A., Lamvik, M. K., Lepault, J., Robertson, J. D., Zeitler, J. D. \& Zemlin, F. (1986). J. Microsc. 141, 385-132.

Cosier, J. \& Glazer, A. M. (1986). J. Appl. Cryst. 19, 105-107.

Crick, F. H. C. \& Magdoff, B. S. (1956). Acta Cryst. 9, 901-908.

de La Fortelle, E. \& Bricogne, G. (1997). Methods Enzymol. 276, 472494. New York: Academic Press.

Diederichs, K., McSweeney, S. \& Ravelli, R. B. (2003). Acta Cryst. D59, 903-909.

Dunlop, K. V., Irvin, R. T. \& Hazes, B. (2005). Acta Cryst. D61, 80-87.

Emsley, P. \& Cowtan, K. (2004). Acta Cryst. D60, 2126-2132.

Ennifar, E., Carpienter, P., Ferrer, J. L., Walter, P. \& Dumas, P. (2002). Acta Cryst. D58, 1262-1268.

Evans, G., Polentarutti, M., Djinovic Carugo, K. \& Bricogne, G. (2003). Acta Cryst. D59, 1429-1434.

Gamblin, S. (2005). Personal communication.

Garman, E. (1999a). Protein Crystallization: Techniques, Strategies, and Tips. A Laboratory Manual, edited by T. Bergfors, ch 17. La Jolla, California: International University Line.

Garman, E. (1999b). Acta Cryst. D55, 1641-1653.

Garman, E. F. \& Doublié, S. (2003). Methods Enzymol, 368, 188-216. Garman, E. F. \& Mitchell, E. M. (1996). J. Appl. Cryst. 29, 584-587. Garman, E. \& Nave, C. (2002). J Synchrotron Rad. 9, 327-328.

Garman, E \& Owen, R. L. (2005). Methods in Molecular Biology, edited by S. Doublié. New Jersey, USA: The Humana Press. (In the press.)

Garman, E. F. \& Schneider, T. R. (1997). J. Appl. Cryst. 30, 211-237.

Garrison, W. (1987). Chem. Rev., 87, 381-398.
Glaeser, R., Facciotti, M., Walian, P., Rouhani, S., Holton, J., MacDowell, A., Celetre, D. \& Padmore, H. (2000). Biophys. J. 78, 3178-3185.

Gonzales, A., Thompson, A. \& Nave, C. (1992). Rev. Sci. Instrum. 63, $1177-1180$.

Gonzalez, A. \& Nave, C. (1994). Acta Cryst. D50, 874-877.

Haas, D. \& Rossmann, M. G. (1970). Acta Cryst. B26, 998-1004.

Halle, B. (2004). Proc. Natl. Acad. Sci. USA, 101, 4793-4798.

Hanson, B. L., Schall, C. A. \& Bunick, G. J. (2003). J. Struct. Biol. 142, 77-87.

Harp, J. M., Timm, D. E. \& Bunick, G. J. (1998). Acta Cryst. D54, 622628.

Helliwell, J. R. (1988). J. Cryst. Growth, 90, 259-272.

Henderson, R. (1990). Proc. R. Soc. London Ser. B, 241, 6-8.

Hendrickson, W. A. \& Ogata, C. M. (1997). Methods Enzymol. 276, 494-523.

Honda, R., Lowe, E. D., Dubinina, E., Skamnaki, V., Cook, A., Brown, N. R. \& Johnson, L. N. (2005). EMBO J. 24, 452-463.

Hope, H. (1988). Acta Cryst. B44, 22-26.

Hope, H. (2001) International Tables for Crystallography: Volume F, Crystallography of Biological Macromolecules, edited by M. G. Rossmann \& E. Arnold, pp. 197-201. Dordrecht: Kluwer Academic Publishers.

Johari, G. P., Hallbrucker, A. \& Mayer, E. (1987). Nature (London), 330, 552-553.

Jones, G. D., Lea, J. S., Symons, M. C. \& Taiwo, F. A. (1987). Nature (London), 330, 772-773.

Juers, D. H. \& Mathews, B. W. (2004). Acta Cryst. D60, 412-421.

Kabsch, W. (1988). J. Appl. Cryst. 21, 916-924.

Kort, R., Komori, H., Adachi, S., Miki, K. \& Ecker, A. (2004). Acta Cryst. D60, 1205-1213.

Kraulis, P. J. (1991). J. Appl. Cryst. 24, 946-950.

Kriminski, S., Caylor, C. L., Nonato, M. C., Finkelstein, K. D. \& Thorne, R. E. (2002). Acta Cryst. D58, 459-471.

Kriminski, S., Kazmierczak, M. \& Thorne, R. E. (2003). Acta Cryst. D59, 697-708.

Kuzay, T. M., Kazmierczak, M. \& Hsieh, B. J. (2001). Acta Cryst. D57, 69-81.

Kwong, P. D. \& Lui, Y. (1999). J. Appl. Cryst. 32, 102-105.

Leiros, H.-K. S., McSweeney, S. \& Smalås, A. O. (2001). Acta Cryst. D57, 488-497.

Matsui, Y., Sakai, K., Murakami, M., Shiro, Y., Adachi, S., Okumura, H. \& Kouyama, T. (2002). J. Mol. Biol. 324(3), 469-481.

McFerrin, M. \& Snell, E. (2002). J. Appl. Cryst. 35, 538-545.

Merritt, E. \& Bacon, D. (1997). Methods Enzymol. 277, 505-524.

Mhaisekar, A., Kazmierczak, M. \& Banerjee, R. (2005). J. Synchrotron Rad. 12, 318-328.

Mitchell, E. M. \& Garman, E. F. (1994). J. Appl. Cryst. 27, 1070-1074.

Müller, R., Weckert, E., Zellner, J. \& Drakopoulos, M. (2002). J. Synchrotron Rad. 9, 368-374.

Murray, J. \& Garman, E. (2002). J. Synchrotron Rad. 9, 347-354.

Murray, J. W., Garman, E. F. \& Ravelli, R. B. G. (2004). J. Appl. Cryst. 37, 513-522.

Murray, J. W., Rudino-Pinera, E., Owen, R. L., Grininger, M., Ravelli, R. B. \& Garman, E. F. (2005). J. Synchrotron Rad. 12, 268-275.

Nave, C. \& Garman, E. F. (2005). J. Synchrotron Rad. 12, 257260.

Nave, C. \& Hill, M. A. (2005). J. Synchrotron Rad. 12, 299-303.

Neutze, R., Wouts, R., der Spoel, D., Weckert, E. \& Hadju, J. (2000). Nature (London), 406, 752-757.

Nicholson, J., Nave, C., Fayz, K., Fell, B. \& Garman, E. (2001). Nuc. Inst. Methods Phys. Res., A467-468, 1380-1383.

O'Neill, P., Stevens, D. L. \& Garman, E. F. (2002). J. Synchrotron Rad. 9, 329-332.

Owen, R. L., Pritchard, M. \& Garman, E. (2004). J. Appl. Cryst. 37, 1000-1003.

Parkin, S. \& Hope, H. (1998). J. Appl. Cryst. 31, 945-953.

Petsko, G. A. (1975). J. Mol. Biol. 96, 381-392. 
Pflugrath, J. W. (2004). Methods Enzymol. 34, 415-423.

Ravelli, R. B., Leiros, H. K., Pan, B., Caffrey, M. \& McSweeney, S. (2003). Structure Fold. Des. 11, 217-224.

Ravelli, R. B. \& McSweeney, S. M. (2000). Structure Fold. Des. 8, 315328.

Ravelli, R. B., Nanao, M. H., Lovering, A., White, S. \& McSweeney, S. (2005). J. Synchrotron Rad. 12, 276-284.

Ravelli, R. B., Theveneau, P., McSweeney, S. \& Caffrey, M. (2002). J. Synchrotron Rad. 9, 355-360.

Riboldi-Tunnicliffe, A. \& Hilgenfeld, R. (1999). J. Appl. Cryst. 32, 1003-1005.

Rodgers, D. W. (1997). Methods Enzymol. 276, 183-202.

Rodgers, D. W. (2001). International Tables for Crystallography: Volume F, Crystallography of Biological Macromolecules, edited by M. G. Rossmann \& E. Arnold, pp. 202-208. Dordrecht: Kluwer Academic Publishers.

Roversi, P., Tsiftsoglou, S. A., Donev, R., Gagg, F., Smith, R. A. G., Sim, R. B., Morgan, B. P. \& Lea, S. M. L. (2004). Abstracts of the XX International Complement Workshop, Molecular Immunology, No. 41, pp. 302-303.

Rubinson, K. A., Ladner, J. E., Tordova, M. \& Gilliland, G. L. (2000). Acta Cryst. D56, 996-1001.

Schiltz, M., Dumas, P., Ennifar, E., Flensburg, C., Paciorek, W., Vonrhein, C. \& Bricogne, G. (2004). Acta Cryst. D60, 10241031.

Skrzypczak-Jankun, E., Bianchet, M. A., Amzel, L. M. \& Funk Jr, M. O. (1996). Acta Cryst. D52, 959-965.

Sliz, P., Harrison, S. C. \& Rosebaum, G. (2003). Structure, 11, 13-19.

Snell, E. H., Judge, R. A., Larson, M. \& van der Woerd, M. J. (2002). J. Synchrotron Rad. 9, 361-367.
Takeda, K., Matsui, Y., Kamiya, N., Adachi, S., Okumura, H. \& Kouyama, T. (2004). J. Mol. Biol. 341, 1023-1037.

Teng, T.-Y. (1990). J. Appl. Cryst. 23, 387-391.

Teng, T.-Y. \& Moffat, K. (2000). J. Synchrotron Rad. 7, 313-317.

Teng, T.-Y. \& Moffat, K. (2002). J. Synchrotron Rad. 9, 198-201.

Thorne, R. E., Stum, J., Kmetko, K., O'Neill, R. \& Gillilan, J. (2003). J. Appl. Cryst. 36, 1455-1460.

Tucker, J. (2005). Personal communication.

Ueno, G., Hirose, R., Ida, K., Kumasaka, T. \& Yamamoto, M. (2004). J. Appl. Cryst. 37, 867-873.

Weik, M., Kryger, G., Schreurs, A. M. M., Bouma, B., Silman, I., Sussman, J. L., Gros, P. \& Kroon, J. (2001). Acta Cryst. D57, 566573.

Weik, M., Ravelli, R. B., Kryger, G., McSweeney, S., Raves, M. L., Harel, M., Gros, P., Silman, I., Kroon, J. \& Sussman, J. L. (2000). Proc. Natl Acad. Sci. USA, 97, 623-628.

Weik, M., Ravelli, R. B., Silman, I., Sussman, J. L., Gros, P. \& Kroon, J. (2001). Protein Sci. 10, 1953-1961.

Weiss, M., Mander, G., Hedderich, R., Diederichs, K., Emler, U. \& Warkentin, E. (2004). Acta Cryst. D60, 686-695.

Weiss, M. S., Panjikar, S., Mueller-Dieckmann, C. \& Tucker, P. A. (2005). J. Synchrotron Rad. 12, 304-309.

Wierenga, R. K., Zeelan, J. P. \& Noble, M. E. (1992). J. Cryst. Growth, 122, 231-234.

Wynne, S. A., Leslie, A. G., Butler, P. J. \& Crowther, R. A. (1999). Acta Cryst. D55, 557-560.

Yeh, J. I. \& Hol, W. G. J. (1998). Acta Cryst. D54, 479-480.

Zaloga, G. \& Sarma, R. (1974). Nature (London), 251, 551-552.

Zwart, P., Banumathi, S., Dauter, M. \& Dauter, Z. (2004). Acta Cryst. D60, 1958-1963. 